\title{
A method to detect p-mode oscillations in solar-like star signals
}

\author{
C. Régulo ${ }^{1,2}$ and T. Roca Cortés ${ }^{1,2}$ \\ 1 Instituto de Astrofísica de Canarias, 38205 La Laguna, Tenerife, Spain \\ 2 Dpto. de Astrofísica, Universidad de La Laguna, La Laguna, 38206, Tenerife, Spain \\ e-mail: trc@astro.iac.es
}

Received 24 April 2002 / Accepted 18 September 2002

\begin{abstract}
A good example of finding a signal buried in noise, a common problem in Astrophysics, is the search for stellar oscillations in the acoustic power spectrum of solar-like stars. In this work it is shown that it is possible to find this type of signal in the power spectrum of stellar oscillations if the peaks are "almost" equally spaced in frequency, as the asymptotic theory for acoustic modes predicts. This signature of the power spectra of the p-mode oscillations in solar-like stars is used to design a method that allows the identification of the signal even when this signal is completely buried in noise, $\sigma_{\text {signal }} / \sigma_{\text {signal+noise }}<1 / 25$.
\end{abstract}

Key words. methods: data analysis - stars: oscillations

\section{Introduction}

The success of helioseismology in probing the interior of the Sun with ever increasing precision fostered the use of seismic techniques to study the interior of other stars. An important part of the future of stellar seismology is related to the study of global p-mode oscillations in solar-like stars. Several attempts at and even claims of the detection of solar-like oscillations has been made in the past (Belmonte et al. 1990; Brown et al. 1991; Pottash et al. 1992; Edmonds \& Cram 1995; Kjeldsen et al. 1995) which have not been fully confirmed yet. More recently, using up to date techniques and new observables, Kjeldsen et al. (1999), Martic et al. (1999), Schou \& Buzasi (2000), Bedding et al. (2001) and Bouchy \& Carrier (2001) have improved the detection possibilities and we are nearer to an indisputable detection. The current space missions COROT (Michel 1998), MONS (Christensen-Dalsgaard, 1998a) and MOST (Matthews 1998) open an extremely interesting future in this field.

However, even from space and having in mind what is observed on the Sun, the signal to noise ratio for solar-like star oscillations will be very small (Christensen-Dalsgaard \& Frandsen 1983; Samadi \& Houdek 2000). Indeed we are trying to detect variations in intensity with respect to the background of one part per million or less. Given this scenario, as was pointed out by Christensen-Dalsgaard (1998b), one of the most important problems in the detection of stellar oscillations is related to data analysis and the process of deciding whether a peak in a crowded spectrum is due to a mode, as opposed to just noise.

Send offprint requests to: C. Régulo, e-mail: crr@astro.iac.es
In this paper we show a method that allows the detection of p-mode oscillation from the power spectrum of solar-like star signals with a very small $S / N$ in the power spectrum of a month of simulated data. The method suggested in Roca Cortés \& Régulo (2001) is based on taking advantage of the "almost" equal frequency spacing that is present in the power spectrum of the solar-like stellar acoustic oscillation signal. This behaviour produces a signature clearly recognizable in the power spectrum of the power spectrum of the stars luminosity or velocity, which allows the recovery of the original signal and the measurement of the frequency modes in cases with very low $S / N$.

The next section describes the basis of the method with a numerically simulated example. In Sect. 3 the method is applied to realistic examples while in Sect. 4 the analysis of a time series of observed earth-based stellar data is shown.

\section{Methodology}

The starting point for the analysis is the power spectrum of the noisy acoustic signal of either luminosity or velocity or any other observable of a star whose acoustic frequencies we are trying to measure.

The idea of the method is to work with the power spectrum of the power spectrum. The frequency range in the power spectrum where the signal is supposed to be buried is selected and its power spectrum is again obtained. Of course the new signal is again a series of peaks equally spaced also buried in noise, but with the advantage that now the signal spans all the transform domain with the first peak being at zero frequency. To find 
the frequency spacing amongst the peaks in such conditions is much easier.

When the correct spacing is found, we go back to find the recovered spectrum of the signal in the following way. In the complex power spectrum of the power spectrum of the signal, all the equally spaced peaks are selected and the inverse Fourier Transform applied. The result will be the recovered power spectrum of the stellar oscillation without noise.

The method can be summarized in the following way. Given an observed signal $m(t)$ :

$m(t)=s(t)+r(t)$

with $s(t)$ being the signal and $r(t)$ being the noise.

Then the FFT is found:

$$
\begin{aligned}
M(v) & =T F[m(t)]=S(v)+R(v), \text { with: } \\
S(v) & =T F[s(t)] \text { and } R(v)=T F[r(t)] .
\end{aligned}
$$

The hypothesis made is that p-modes present equally spaced frequency peaks showing a signature:

$S(v)=\sum_{n=n_{i}}^{n=n_{j}} \delta\left(v-n v_{0}\right)$

where $n_{i}, n_{j}$ are integers such that $n_{i}<n_{j}, n_{i} v_{0}=v_{1}$ and $n_{j} v_{0}=$ $v_{2}$ with $v_{0}$ been the frequency spacing. Obviously $v_{0}, v_{1}$ and $v_{2}$ are unknown in general. However, they can be scaled from the known solar values (Kjeldsen \& Bedding 1995) and even can be predicted from theory. Therefore, assuming $v_{1}$ and $v_{2}$ are known, a part of $M(v)$ with $v_{1} \leq v \leq v_{2}$ is selected and its Fourier Transform obtained, so:

$$
\begin{aligned}
T F[M(v) \cdot H(v)]= & {[T F(S(v)) \cdot T F(H(v))] } \\
& +[T F(R(v)) \cdot T F(H(v))],
\end{aligned}
$$

with:

$H(v)=1, v_{1} \leq v \leq v_{2}$

$H(v)=0$, otherwise.

The first term on the right hand side is,

$$
\frac{1}{v_{0}} \sum_{n=-\infty}^{n=\infty} \delta\left(f-\frac{n}{v_{0}}\right) \cdot 2 v_{\mathrm{t}} \frac{\sin \left(2 \pi v_{\mathrm{t}} f\right)}{2 \pi v_{\mathrm{t}} f} \mathrm{e}^{-2 \pi i f v_{z}},
$$

being $v_{\mathrm{t}}=\left(v_{2}-v_{1}\right) / 2$ and $v_{z}=v_{1}+v_{\mathrm{t}}$.

Now, an iterative method is used to find out if there is signal only at the bins spaced by $v_{0}^{-1}$. Using several values of $v_{0}$ that cover a flexible range around the expected value, with a step of $1 \mathrm{bin}$, the power at the bins with $f=n v_{0}^{-1}$ are statistically studied to search for the best value that fits the best signature assumed above. The success in this process obviously depends on the $S / N$ ratio present in the data.

Once the spacing is found, the function $T F[M(v) \cdot H(v)]$ is filtered by keeping only the bins that are a multiple of $v_{0}^{-1}$ while setting all others to null. To this function the inverse Fourier Transform is applied, that is:

$T F^{-1}\left(\sum_{n=-\infty}^{n=\infty} A_{n} \delta\left(f-\frac{n}{v_{0}}\right) \cdot \mathrm{e}^{2 \pi i f v_{z}}\right)$, and when $A_{n}=1$, the result is exactly:

$\sum_{n=-\infty}^{n=\infty} \delta\left[\left(v+v_{z}\right)-n v_{0}\right]$

however as $A_{n} \neq 1$ in general, some noise appears in the recovered signal whose level depends on their values.

Let's give a practical example to illustrate how the method works. A signal of 182 days at $60 \mathrm{~s}$ sampling has been generated. The signal consists of 30 coherent sine waves, with the same amplitudes (unity) and random phases, plus white noise (having zero average and a $\sigma \approx 100$ ); the frequency of the first sine wave is $2.1 \mathrm{mHz}$ and the frequency spacing is $0.1267 \mathrm{mHz}$. Thus the $S / N$ defined as $\sigma_{\text {signal }} / \sigma_{\text {noise+signal }}$ is equal to 0.039 , although for any sine wave the $S / N \approx 1 / 100$. Notice that this $S / N$ definition is made in time-space but this could be translated to frequency-space using Parseval's theorem; however, it is much more difficult to calculate, especially when the signals are so small that the real peaks interfere with the noise ones in either a constructive or destructive way, yielding a great variety of peak amplitudes (see Fig. 1). In Fig. 1 (top) a piece of the power spectrum of the noisy signal is plotted and in Fig. 1 (second plot), the same piece of the power spectrum of the signal is shown but without noise. With this level of noise, to find the signal buried in it is almost impossible and even to try to find the spacing among the peaks is very difficult; moreover, in a real case, we are not sure where to look for it.

The analysis starts by selecting a sub-spectrum, say between $v_{1}=2.2 \mathrm{mHz}$ and $v_{2}=5.5 \mathrm{mHz}$, where we asume the signal is present; notice that is not precisely so. First, the power spectrum of the sub-spectrum is done. From this power spectrum, the frequency spacing of $v_{0}=0.1267 \mathrm{mHz}$ is detected without problems in this particular case. Moreover, this is the only one detected, apart from its harmonics, by analysing the power at the bins with exact multiples of $v_{0}^{-1}$. The original signal is then recovered by selecting in the complex power spectrum of the sub-spectrum all bins spaced $0.1267^{-1} \mathrm{mHz}^{-1}$ and doing the inverse Fourier Transform for this selected signal as explained before.

Looking at the recovered power spectrum, the peaks are clearly visible above the noise; however, we can set some general criteria, such as: peaks are selected as signal if they have a power greater than $50 \%$ of the highest peak and if their phases are close to 0.0 . With these criteria we try to avoid the selection of any peak resulting from noise, although some signal peaks can be lost. As it can be see in Fig. 1 (third plot), the signal is recovered in a perfect way. All signal peaks have been recovered with a precision in frequency better than the spectral resolution $(60 \mathrm{nHz})$, and no peak coming from noise appears in the recovered signal.

Furthermore, if we assume that we have got a wrong spacing, say $v_{0}=0.1260 \mathrm{mHz}$, and it is used to recover the original signal, the result is plotted in Fig. 1 (bottom). The level of noise is now as low as in the case where the signal is well recovered, because the same percentage of bins has been set to zero, but no signal appears above the noise. Only when the real peaks of signal are selected among the noise do the phases have the correct relation to reconstruct the signal; otherwise, a noise spectrum is recovered. 

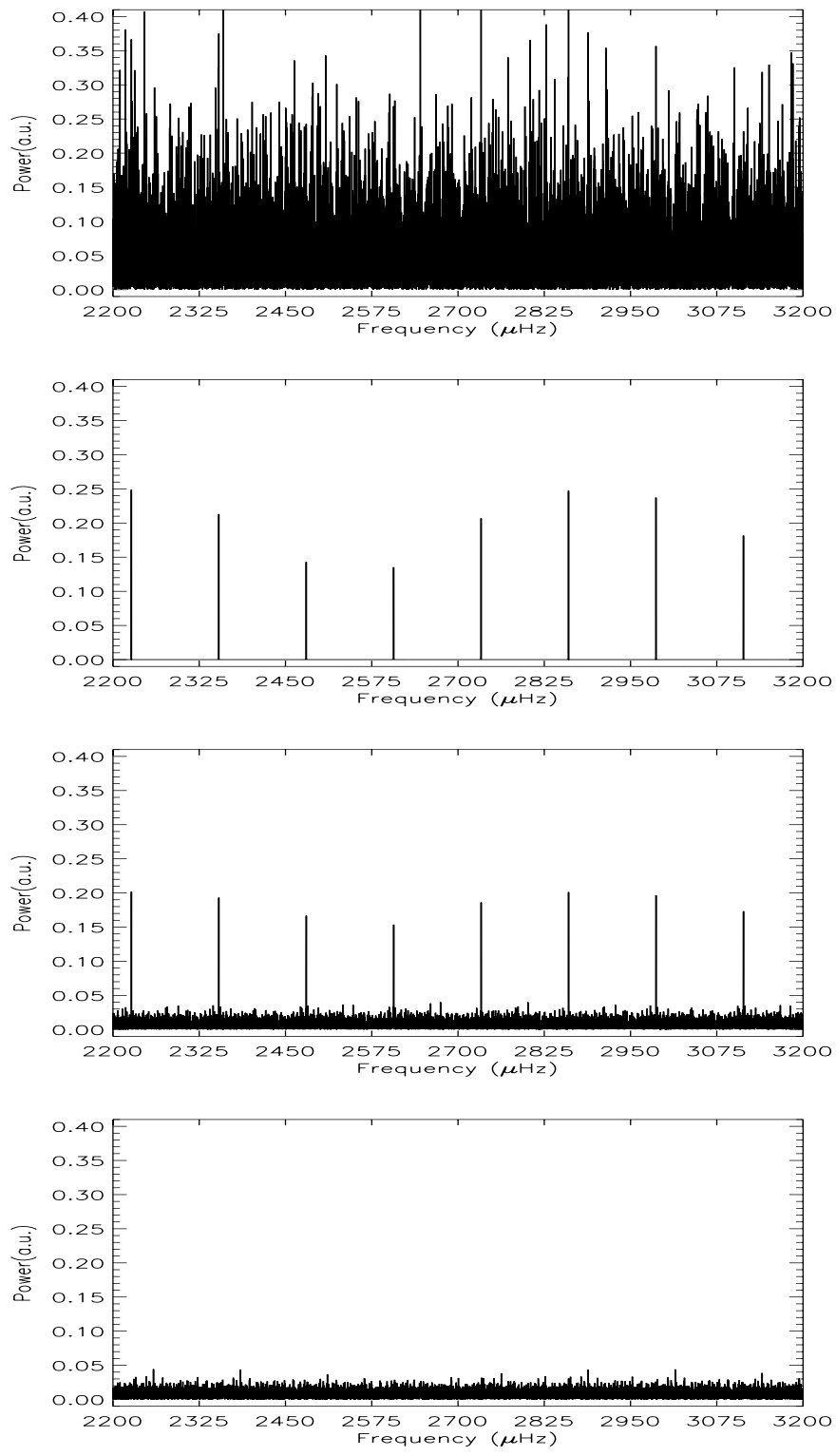

Fig. 1. From top to bottom, first plot: a piece of the power spectrum of a 182 day generated signal, composed of 30 equidistant frequencies, that simulate the signal, plus white noise. Second plot: the same piece of the power spectrum of the simulated signal without noise. Third plot: the same piece of the power spectrum of the recovered signal after the method explained in the text has been applied. Fourth plot: the same piece of the power spectrum of the recovered signal when a wrong spacing is used.

Another fact to be taken into account is that when only one spacing is present in the data, the signal is better recovered if the length of the sub-spectrum chosen is a multiple of the spacing, because in that case, the signal we are working with is truly a periodic signal in the FFT sense. It is important to note that the method is also able to recover more than one spacing provided the spacings are present in the data, as was already shown in Roca Cortés \& Régulo (2001).

A problem will arise if the frequency spacing $v_{0}$ is not exactly constant because this will break our main assumption. Therefore, two related parameters appear in the method: the

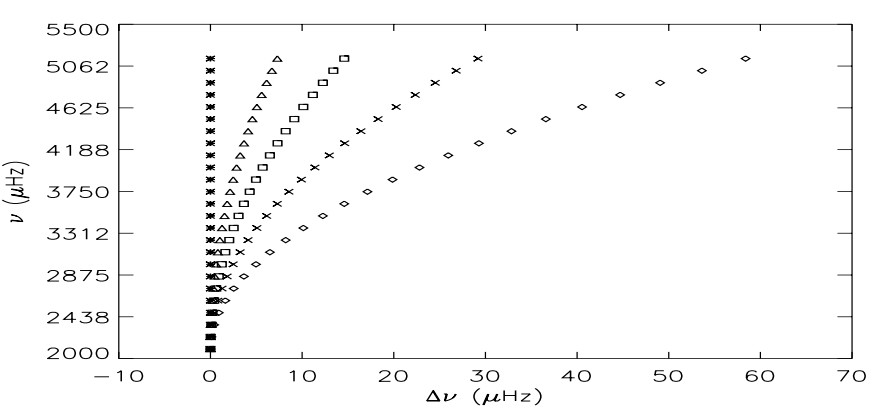

Fig. 2. "Echelle diagram" for the frequencies generated in Sect. 2. In this plot $v=2100+v_{0} \cdot n$, where $v_{0}=126.7 \mu \mathrm{Hz}$ and $n$ is the signal's peak number. $\Delta v=\left(\Delta v_{0} \cdot n / M\right) \cdot n$, being $M$ the total number of peaks. The asterisks show the case with no departure from the regular pattern and correspond to $\Delta v_{0}=0.0 \% v_{0}$, the triangles correspond to $\Delta v_{0}=0.25 \% v_{0}$, squares for $\Delta v_{0}=0.50 \% v_{0}, \mathrm{X}$ for $\Delta v_{0}=1.0 \% v_{0}$ and diamonds for $\Delta v_{0}=2.0 \% v_{0}$.

Table 1. Uniform frequency spacing (in $\mathrm{mHz}$ ) found in 15 simulated time series with varying $S / N$ and frequency spacing $\left(v_{0}=\right.$ $0.1267 \mathrm{mHz}$ ).

\begin{tabular}{c||c|c|c}
\hline \hline \multicolumn{1}{c||}{} & \multicolumn{3}{c}{$\sigma_{\text {signal }} / \sigma_{\text {signal+noise }}$} \\
\hline$\Delta v_{0} / v_{0}(\%)$ & 0.077 & 0.052 & 0.038 \\
\hline 0.00 & 0.1267 & 0.1267 & 0.1267 \\
0.25 & 0.1268 & 0.1270 & - \\
0.50 & 0.1272 & 0.1268 & 0.1265 \\
1.00 & 0.1273 & 0.1271 & - \\
2.00 & 0.1295 & - & - \\
\hline
\end{tabular}

$S / N$ level and the departure of the frequency spacing $v_{0}$ from constant.

In order to get a quantitative measure of the relative importance of such parameters, the method has been applied to 15 time series similar to the one already used, changing both the $S / N$ level and the uniformity in the frequency spacing. The spacing simulated is now a linear departure from uniform in the following sense: $v_{0}^{\prime}=v_{0}+\Delta v_{0} \cdot n / M$, where $\Delta v_{0}$ measures the departure from uniformity, $n$ is the signal's peak number and $M$ is the total number of signal peaks. Time series with $\Delta v_{0}=0.0 \% v_{0}, 0.25 \% v_{0}, 0.50 \% v_{0}, 1.0 \% v_{0}$ and $2.0 \% v_{0}$ have been generated, each one with 3 different levels of noise, so that the $S / N=0.077,0.052$ and 0.038 . The departure from an exact frequency spacing in the new generated examples are plotted in Fig. 2 in an "echelle diagram".

The method is applied to these new 15 series searching for uniform frequency spacings in the same way as explained before. Results are shown in Table 1, where the values found can be seen in each simulated case. It is clear from theses results that the smaller the $S / N$ level of our observations, the smaller the departure from an uniform frequency spacing the method will be able to accept.

\section{Analysis of solar-like p-modes in simulated data}

To see the performance of the method in more realistic cases than the example presented in the section above, two examples have been developed following the characteristics and conditions that are expected for solar-like stellar oscillations. 
One month of data has now been used in the examples because this is an expected period to be obtained from current space missions and it is a reasonable maximum period to be achieved from a network of two or three medium size ground based telescopes. For each example the data has been simulated with and without gaps, to simulate observation from groundbased observatories and from space.

\subsection{First example}

In this first example data with $60 \mathrm{~s}$ sampling has been simulated in the following way: the signal consists of 20 sine waves representing $\ell=0 \mathrm{p}$-modes starting at $1.7450 \mathrm{mHz}$ and with a frequency spacing of $0.1252 \mathrm{mHz}$; added to this signal, 40 sine waves in couples, separated by $800 \mathrm{nHz}$ (simulating a frequency splitting of $400 \mathrm{nHz}$ ), represent $\ell=1 \mathrm{p}$-modes starting at $1.7926 \mathrm{mHz}$, with the same frequency spacing used for the $\ell=0$ case; further, added to this signal, another 60 sine waves in triplets, separated by $810 \mathrm{nHz}$ (simulating a splitting of $810 \mathrm{nHz}$ between even $m$ modes) represent $\ell=2$ p-modes, starting at $1.7300 \mathrm{mHz}$ and with the same frequency spacing. Thus the signal simulates the observed characteristics of the solar signal for $\ell=0, \ell=1$ and $\ell=2$ p-modes. The amplitudes of the sine waves are all equal to unity but their phases are set at random; to this signal, white noise is added with a $\sigma_{\text {signal }} / \sigma_{\text {signal+noise }} \approx 0.098$. In the Fig. 3 (top) a slice of the power spectrum of the signal plus noise is shown while in the central plot the power spectrum of the signal only is plotted.

In the process of analysing such simulated time series, we assume that the signal could be around $3.0 \mathrm{mHz}$ and the search is done between $2.0 \mathrm{mHz}$ and $4.0 \mathrm{mHz}$. Notice that in this range, only 16 peaks of each $\ell$ value are present. The first step is to calculate the power spectrum of the sub-spectrum selected and look for any frequency spacing present. The ability to find the frequency spacing depends on the signal to noise ratio. In the present case the spacing of $0.1252 \mathrm{mHz}$ is found without difficulty. With this spacing we went back to recover the spectrum of the signal as it is explained in the previous section. The recovered signal is shown in Fig. 3 (bottom).

In the recovered spectrum the peaks are accepted as signal peaks if they follow the criteria given in the previous section: their power must be $\geq 50 \%$ of the highest peak and their phases $\approx 0.0$. Following these criteria $15 \ell=1$ and $15 \ell=2$ p-modes have been recovered.

The $\ell=0 \mathrm{p}$-modes are not recovered at the level the others are; as it can be seen in Fig. 3 (bottom), they appear in the recovered signal but with an amplitude so small that are not selected as signal peaks because of the criteria used. However it is interesting to notice that in Fig. 3 (middle plot) where the simulated signal is shown without noise, the $\ell=0$ p-modes appear smaller than the $\ell=1$ and $\ell=2 \mathrm{p}$-modes, although they were originally generated with the same amplitude. This is probably a result of the fact that the $\ell=1$ and $\ell=2$ p-modes have structure (more than one peak) and some kind of constructive interference enhances the amplitude of these modes. No noise peak is selected as signal. From the $15 \ell=1 \mathrm{p}$-modes recovered, 11 are recovered as doublets with the exact simulated
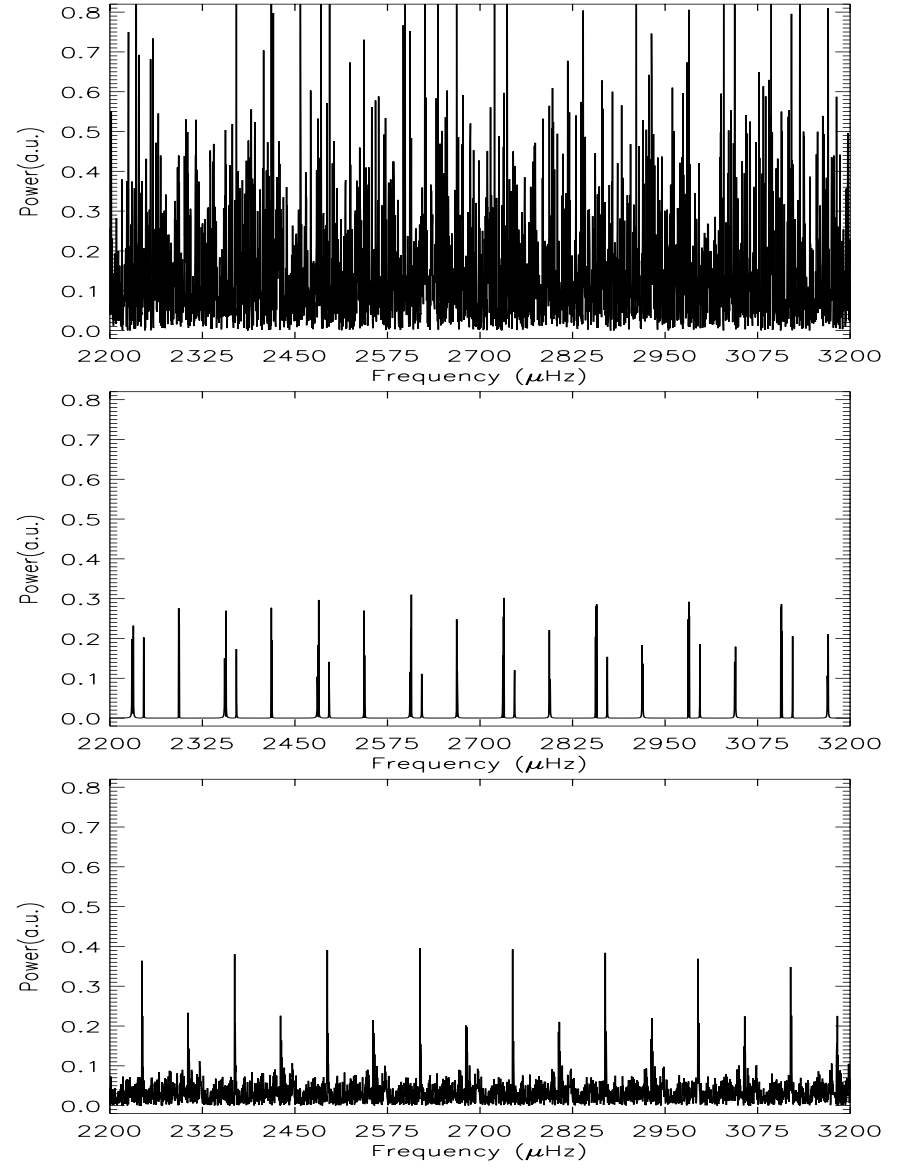

Fig. 3. Top plot: a piece of the power spectrum of a month simulated signal; the signal is composed of 120 sine waves representing some $\ell=0, \ell=1$ and $\ell=2$ p-modes plus white noise. Middle plot: the same piece of the power spectrum of the simulated signal but without noise. Bottom plot: the same piece of the recovered power spectrum from the top spectrum using the method described in the text.

frequencies; in the case of the 4 singlets only one of the two $m$ components is obtained. As far as the $15 \ell=2 \mathrm{p}$-modes is concerned, 13 have been recovered as singlets and 2 as doublets. For 6 of them, the recovered peak is the central one $m=0$, for the rest, only one of the $m$ components is recovered.

Although only one month of data has been generated and with a signal completely buried in noise, the obtained results are quite good. Most of the signal peaks have been recovered, even with their splitting, without any noise peaks among them. Changing the length and position of the selected spectrum does not improve the results much, although using the "known" inputs, more signal is recovered.

Finally, using the same signal, gaps have been introduced to simulate observation from Earth. Introducing gaps decreases the signal to noise ratio. With the use of $25 \%$ of zeros every 24 hours in a random way, slightly worse results are obtained; however, the signal spacing $0.1252 \mathrm{mHz}$ still is obtained from the power spectrum of the power spectrum between $2.0 \mathrm{mHz}$ and $4.0 \mathrm{mHz}$. Again, $15 \ell=1 \mathrm{p}$-modes are recovered, 10 perfect doblets and 5 singlets, but only $8 \ell=2$ p-modes are obtained, all of them as singlets; in all cases, the recovered peak is the $m=0$, given a perfect frequency for the mode. 


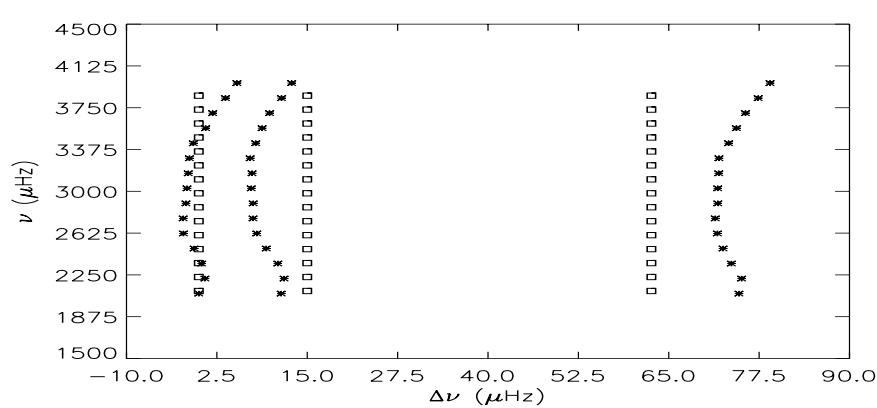

Fig. 4. Echelle diagram for the frequencies of the examples in Sect. 3.1 (squares) and Sect. 3.2 (asterisks) where the deviation of the observed modes from an equally spaced pattern is clearly visible. In this plot, $v=v_{\text {ini }}+v_{0} \cdot n$; for the squares $v_{\text {ini }}=2105.6 \mu \mathrm{Hz}, v_{0}=125.2 \mu \mathrm{Hz}$ and for the asterisks $v_{\text {ini }}=2082.10 \mu \mathrm{Hz}, v_{0}=134.9 \mu \mathrm{Hz}$, and $n$ is the signal's peak number; $\Delta v=v_{\text {real }}-v, v_{\text {real }}$ being the value of each frequency in the power spectrum.

\subsection{Second example}

This is a more realistic case where one month of actually observed data from the GOLF experiment onboard SOHO (Gabriel et al. 1995) has been used. To this real signal, white noise has been added to simulate stellar conditions. In this example $\sigma_{\text {signal }} / \sigma_{\text {signal+noise }} \approx 0.158$, which is the lowest signal that can be recovered under these conditions. The problem now is that the frequencies in the power spectrum of this signal are not exactly equally spaced and as already stated, the method proposed is based on this hypothesis. See Fig. 4 for an echelle diagram of the frequencies used in the already simulated example (Sect. 3.1) and the observed GOLF ones where the difference in the uniformity of the spacings is very clear. In this realistic case, the amplitudes are higher for the $\ell=0$ and $\ell=1$ p-modes, keeping the average amplitude ratio observed in the Sun. Moreover, the amplitudes are maximum around $3.2 \mathrm{mHz}$, falling rapidly on both sides; see Fig. 5 (middle) where the true power spectrum of the observed GOLF data is shown.

Therefore, in the analysis of this spectrum, in trying to minimize the problem instead of using the power spectrum of the signal directly, an average of 2 spectra of half length is used. The idea is to use a coarser frequency bin in order to move our spectrum signal nearer to one with an equal spacing interval in the power spectrum. The price we pay, of course, is worse resolution in frequency, which is now of $770 \mathrm{nHz}$. See Fig. 5 (top).

The average power spectrum of the 2 sub-series is analysed between $2.6 \mathrm{mHz}$ and $3.7 \mathrm{mHz}$. In this frequency range $8 \ell=0$, $8 \ell=1$ and $8 \ell=2$ p-modes are present. From the power spectrum of this selected sub-spectrum, a spacing of $0.1349 \mathrm{mHz}$ is obtained. With this spacing, the recovered spectrum is calculated and following the same criteria as in previous examples, all the $8 \ell=0$ and the $8 \ell=1 \mathrm{p}$-modes have been recovered, but not the $\ell=2$ p-modes because of their lower amplitudes. See Fig. 5 (bottom).

From the $\ell=0$ p-modes recovered, only one has a difference in frequency, with the real one, bigger than the spectral resolution. From the $\ell=1 \mathrm{p}$-modes, all recovered as singlets because of the spectral resolution, 4 of them have differences
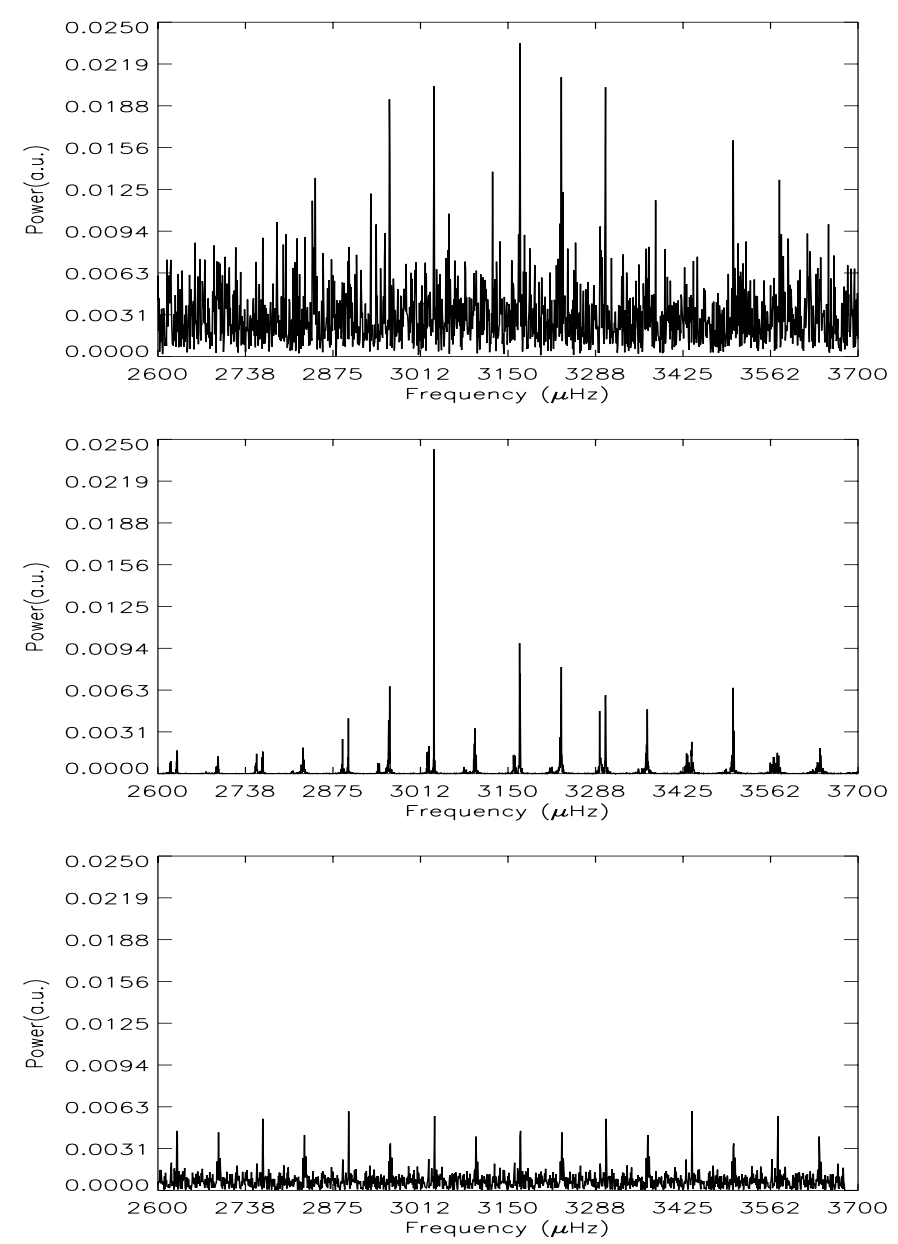

Fig. 5. Top plot: a piece of the mean power spectrum of one month of GOLF data cut into two pieces plus white noise. Middle plot: the same piece of the power spectrum of the GOLF data but without the added noise. Bottom plot: the same piece of the recovered power spectrum from the top spectrum using the method described in the text.

respect to the frequencies of real peaks of around $1000 \mathrm{nHz}$, slightly above the spectral resolution.

When gaps are added to the signal, produced as in the previous example every 24 hours in a random way, the decrease in the signal to noise ratio prevents us from finding the spacing if the percentage of zeros are between $12 \%$ and $25 \%$. With $12 \%$ zeros, the $0.1349 \mathrm{mHz}$ spacing is again found but when the spectrum is recovered with this spacing, only the $\ell=0 \mathrm{p}$ modes are found. However, they are all recovered and with a precision better than the spectral resolution and, very importantly, no peaks from noise are selected as signal.

These results are very interesting because the example reproduces the expected stellar data conditions with frequencies not exactly equally spaced and with a poor signal to noise ratio. Even in these conditions a significant percentage of frequencies has been well recovered and, more importantly, no peaks from noise have been selected.

\section{Analysis of observed earth-based stellar data}

In order to verify the performance of the method with even more real data, we have used observations from $\alpha$ Cen A kindly 
provided by Hans Kjeldsen and Tim Bedding (Kjeldsen et al. 1999). The data were collected over six nights in April 1995 from two sites (Siding Spring Observatory in Australia and the Europen Southern Observatory at La Silla in Chile) with an overall duty cycle of $75 \%$. They measured Balmer line equivalent widths to look for solar-like oscillations and granulation.

We started using the power spectrum of the data (produced by Kjeldsen et al. 1999). Although the true resolution of the data is only $2000 \mathrm{nHz}$, the data has been padded with zeros to yield a spectral resolution of $100 \mathrm{nHz}$. From the power spectrum of this power spectrum we tried to find the acoustic mode signature in the frequency range between 1.6 and $2.8 \mathrm{mHz}$, where the signal is expected to have its maximum amplitude according to the mass and effective temperature of the star (Kjeldsen \& Bedding 1995).

Applying the method described in this frequency range, only two possible frequency spacings are barely found, one at $0.1057 \mathrm{mHz}$ and a second one at $0.1394 \mathrm{mHz}$. Although the first one approximately agrees with the expected value predicted by the stellar models (Morel et al. 2000), no clear signals are recovered from it when the inverse Fourier Transform was applied, so the recovered power spectrum looks like the one shown in Fig. 1 (bottom). Thus, if any signal is present in the data, it is too weak compared to noise to be clearly detected using our method.

Trying to see whether the spacing found could be produced by a combination of noise and the observing window function, a pure noise signal was simulated using the window function and the weights of the real signal, and its power spectrum was obtained with a frequency sampling of $500 \mathrm{nHz}$ in a weighted iterative sine wave fitting procedure. However, in the analysis of this power spectrum using our method, no frequency spacing was found.

To search for the limits of detection of the method in this particular case, a known signal was injected into the observed data. The injected signal has the following characteristics: 12 coherent sine waves, with the first one at $1.6 \mathrm{mHz}$ and having a frequency spacing of $0.1020 \mathrm{mHz}$. The window function and the weight values of the observed series were used to produce this simulated signal. The amplitude of each sine wave was left free to determine a minimum value for the detection method to recover the injected signal. The result found is that it must have an amplitude of at least $6 \mathrm{ppm}$ (parts per million).

It is interesting to compare these results with the ones obtained by Bouchy \& Carrier (2001). Using the CORALIE spectrograph on the $1.2 \mathrm{~m}$ Swiss telescope at the ESO observatory during 5 nights of observation, the authors claim a clear detection of $\mathrm{p}$-mode oscillations in $\alpha$ Cen A. The mean spacing found is $0.1057 \mathrm{mHz}$ and the mean amplitude of the modes is about $0.3 \mathrm{~m} \mathrm{~s}^{-1}$. Such velocity amplitude can be translated into intensity variations ( $\mathrm{ppm})$ given the effective temperature of the star (Kjeldsen \& Bedding 1995). Therefore, assuming $T_{\text {eff }}=5790 \mathrm{~K}$ for $\alpha$ Cen A (Morel et al. 2000) the $\approx 0.3 \mathrm{~m} \mathrm{~s}^{-1}$ velocity amplitude obtained by Bouchy and Carrier corresponds to $\approx 5 \mathrm{ppm}$. Although the data we have analysed, obtained by observing the equivalent widths of some Balmer lines, are slightly more sensitive than intensity variation, the
5 ppm could be somewhat higher and thus be close to the required signal level we would need to detect the oscillation.

\section{Conclusions}

A method to detect the frequencies of several periodic functions of a signal buried in noise has been developed. The signal must have only one specific characteristic: the periodic functions that make up the signal must have frequencies "almost" equally spaced. Some previous knowledge about the signal is important to apply the method. First, it is necessary to have an idea about the frequency range where the signal appears and, second, it is important to know the order of magnitude of the frequency spacing. These conditions are met in the stellar oscillations topic for which the method has been developed.

The method works better when the frequencies are equally spaced. In this case, the method is able to recover almost completely the power spectrum of the signal even with a $\sigma_{\text {signal }} / \sigma_{\text {noise }}<1 / 25$. Even if the frequencies depart from this exact signature, the method still works well, although some precision is lost in the recovered frequencies because of the necessity of increasing the frequency bin. Obviously, the $S / N$ ratio and the 'departure' from an even or regular frequency spacing are parameters that play a key role in the success of the method.

Two realistic simulated series of data and one real observed series have been used to analyse the performance of the method. It is interesting to note that in the first example analysed, where all the $\ell$ p-modes were generated with the same amplitude, the $\ell=0 \mathrm{p}$-modes were not recovered whereas almost all the $\ell=1$ and $\ell=2$ p-modes were obtained. It is clear that having some structure, such as more than one peak, helps the process of recovering the signal. However, in the second example the $\ell=0 \mathrm{p}$-modes are well recovered, and they are the only recovered peaks when gaps are introduced, due to their higher amplitude, whereas the $\ell=2$ p-modes are not recovered, given their structure, because of their low amplitude.

In both examples all frequencies of peaks are recovered with a precision of one frequency bin, and in some cases even their splitting is well recovered. Even just the possibility of obtaining the spacing is interesting enough, because it is capable of giving an important restriction to the physical properties of the stars (Guenther 1998) allowing one to restrict the models and to distinguish among different equally possible models.

The proposed procedure is also robust enough (it does not confuse real from noise peaks) to be used to analyse stellar oscillation signals, as has been proved from the $\alpha$ Cen A data analysis. However, the present data seem to be at the limit of the signal to noise ratio to allow clear identification of the individual p-mode frequency oscillations.

This method could be also useful to look for g-modes in solar data series, especially in the asymptotic regime where the modes present regular signatures (now in period rather than frequency), even though the signal to noise is predicted to be very low (Kumar 1997). Such a possibility is being explored and is under development at the moment. 
Acknowledgements. The authors acknowledge M. Collados for many enlightening discussions, H. Kjeldsen and T. Bedding for the use of their $\alpha$ Cen A data and R. García Bustinduy for the calibration of the GOLF data used. We would like also to thank all our colleages: scientists, engineers and technicians involved with the GOLF project.

\section{References}

Bedding, T. R., Butler, R. P., Kjeldsen, H., et al. 2001, ApJ, 549, L105 Belmonte, J. A., Jones, A. R., Pallé, P. L., \& Roca Cortés, T. 1990, Ap\&SS, 169, 77

Bouchy, F., \& Carrier, F. 2001, A\&A, 374, L5

Brown, T. M., Gilliland, R. L., Noyes, R. W., \& Ramsey, L. W. 1991, ApJ, 368, 599

Christensen-Dalsgaard, J., \& Frandsen, S. 1983, Sol. Phys., 82, 469

Christensen-Dalsgaard, J. 1998a, Proceedings of the First MONS Workshop, Aarhus, Denmark, ed. H. Kjeldsen, \& T.R. Bedding, Aarhus Universitet, 17

Christensen-Dalsgaard, J. 1998b, Proceedings of the SOHO 6/GONG 98 Workshop, Structure and Dynamics of the Interior of the Sun and Sun-like Stars, Boston, USA ESA SP-418, 17

Edmonds, P. D., \& Cram, L. E. 1995, MNRAS, 276, 1295

Gabriel, A. H., Grec, G., Charra, J., et al. 1995 Sol. Phys., 162, 61

Guenther, D. B. 1998, Proceedings of the SOHO 6/GONG 98 Workshop, Structure and Dynamics of the Interior of the Sun and Sun-like Stars, Boston, USA ESA SP-418, 375
Kjeldsen, H., Bedding, T. R., Viskum, M., \& Frandsen, S. 1995, AJ, 109,1313

Kjeldsen, H., \& Bedding, T. R. 1995, A\&A, 293, 87

Kjeldsen, H., Bedding, T. R., Frandsen, S., \& Dall, T. H. 1999, MNRAS, 303, 579

Kumar, P. 1997, Sounding Solar and Stellar Interiors, Nice, France, ed. J. Provost, \& F.X. Schmider, IAU Symp. 181, 287

Martic, M., Schmitt, J., Lebrun, J. C., et al. 1999, A\&A, 351, 993

Matthews, J. M. 1998, Proceedings of the SOHO 6/GONG 98 Workshop, Structure and Dynamics of the Interior of the Sun and Sun-like Stars, Boston, USA ESA SP-418, 395

Michel, E. 1998, Proceedings of the SOHO 6/GONG 98 Workshop, Structure and Dynamics of the Interior of the Sun and Sun-like Stars, Boston, USA ESA SP-418, 399

Morel, P., Provost, J., Lebreton, Y., Théveniu, F., \& Berthomieu, G. 2000, A\&A, 363, 675

Pottash, E., Butcher, H. R., \& van Hooesel, F. H. J. 1992, A\&A, 264, 138

Roca Cortés, T., \& Régulo, C. 2001, Proceedings of the SOHO 10/GONG 2000 Workshop, Helio- and Asteroseismology at the Dawn of the Millennium, Sta. Cruz de Tenerife, Spain, ESA SP464,447

Samadi, R., \& Houdek, G. 2000 Proceedings of the Third MONS Workshop, Aarhus, Denmark, ed. T.C. Teixeira, \& T.R. Bedding, Aarhus Universitet, 27

Schou, J., \& Buzasi, D. L. 2000, A\&AS, 197, 4604 